\title{
Successes and failures: what did we learn from recent first-line treatment immunotherapy trials in non-small cell lung cancer?
}

\author{
Jordi Remon ${ }^{1,2}$, Benjamin Besse $e^{1,3}$ and Jean-Charles Soria ${ }^{3^{*}}$
}

\begin{abstract}
Immune checkpoint inhibitors have significantly modified the therapeutic landscape of advanced non-small cell lung cancer in second-line settings, with a more recent advancement in first-line settings. Given the superior outcome with pembrolizumab as an upfront strategy, PD-L1 status should now be considered a new reflex biomarker to guide first-line treatment in patients with advanced non-small cell lung cancer. Improved responses have also been reported with the combination of immune checkpoint inhibitors and chemotherapy as the first-line treatment; however, this strategy has not yet been validated by phase III trial data and its interplay with PD-L1 status still requires clarification.

Herein, we review the contradictory results of recent phase III trials with immune checkpoint inhibitors in the first-line setting, the potential reasons for such discrepancies, and some of the remaining points of discussion related to the positioning of immune checkpoint inhibitors in the first-line therapy of non-small cell lung cancer.
\end{abstract}

Keywords: Immunotherapy, Pembrolizumab, Nivolumab, PD-1, PD-L1, First-line, Non-small cell lung cancer

\section{Background}

First-line platinum-based chemotherapy is the standard of care in the majority of patients with advanced non-small cell lung cancer (NSCLC) without comorbidities and with an optimal performance status [1]; this excludes patients with oncogenic driver alterations, such as the epidermal growth factor receptor $(E G F R)$ mutation (in almost $50 \%$ of patients of Asian ethnicity compared to $15 \%$ in the Caucasian population [2]) or the anaplastic lymphoma kinase $(A L K)$ re-arrangement (in 5\% patients independently of ethnicity [3]), who can be treated with tyrosine kinase inhibitors. However, even in the era of maintenance therapy, platinum-based chemotherapy only results in a median progression-free survival (PFS) of approximately 6 months and a response rate (RR) of approximately 30\% [1]. Therefore, significant advances are eagerly awaited.

\footnotetext{
* Correspondence: jean-charles.soria@gustaveroussy.fr

The original version of this article was revised: published text contained several minor typos.

${ }^{3}$ University Paris-Sud, Orsay, France

Full list of author information is available at the end of the article
}

A deeper understanding of tumor-immune interactions and development of immune checkpoint inhibitors has dramatically changed the therapeutic landscape of NSCLC and other malignancies. The immune system recognizes and is poised to eliminate cancer [4]. Immune checkpoints refer to a variety of inhibitory pathways that are crucial in regulating the duration and amplitude of physiological immune responses in peripheral tissues in order to minimize collateral tissue damage [5]. However, these immune checkpoint pathways can be co-opted by cancer cells, thus circumventing immune destruction [4]; indeed, this is a hallmark of cancer [6]. In NSCLC, expression of programmed death ligand-1 (PD-L1, B7-H1) reflects an immune-active microenvironment and is a mechanism designed to evade elimination by the immune system [7]. Exhausted T-cells in the microenvironment show overexpression of programmed cell death protein 1 (PD-1), which binds to PD-L1 and decreases effector cytokine production and cytolytic activity, leading to the failure of cancer elimination [8]. This knowledge has prompted the development of immune checkpoint inhibitors - different 
monoclonal antibodies that bind either to PD-1 or PD-L1 and hamper immune evasion - as new treatment strategies in advanced NSCLC.

Four randomized phase III trials have reported a statistically significant improvement in overall survival (OS) with immune checkpoint inhibitors compared with docetaxel in patients with platinum-refractory advanced NSCLC. These are the CheckMate 017 trial in patients with squamous NSCLC [9]; the CheckMate 057 trial in patients with non-squamous NSCLC (both trials testing nivolumab, a monoclonal-antibody antiPD-1) [10]; the KEYNOTE-010 phase II/III trial with pembrolizumab [11], which also binds to PD-1 (inclusion restricted to patients with at least 1\% PD-L1 expression on tumor cells); and the OAK trial with atezolizumab [12], a monoclonal antibody against PD-L1. Of note, in the KEYNOTE-010 study [11], the magnitude of benefit with pembrolizumab was correlated to PD-L1 expression, showing increased benefit in patients with tumors with strong PD-L1 expression (defined as expression on at least $50 \%$ of tumor cells, regardless of the staining intensity with the 22C3 clone) [11]. On the basis of these trials, the US Food and Drug Administration (FDA) and the European Medicines Agency (EMA) approved nivolumab and pembrolizumab as second-line therapies, with the latter being restricted to tumors expressing PD-L1. The FDA has also recently approved atezolizumab for the management of previously treated patients with advanced NSCLC. In the absence of head-to-head comparisons or clear biological differences between these agents, and no significant differences in toxicity profile (except a slight increase in immune-related adverse events (AEs) and pneumonitis with anti-PD-1 inhibitors) [13], recommendation of a given treatment over another is not yet possible.

The 3-year OS of patients with advanced NSCLC treated with these drugs after failure with platinum-based chemotherapy is approaching 20\% [14]. This response, along with an improved safety profile, has prompted increasing interest in testing these agents in the first-line setting.

\section{Anti-PD-1 antibodies as a single agent in patients with PD-L1-positive NSCLC Pembrolizumab}

The phase III KEYNOTE-024 trial comprises patients with advanced and strongly PD-L1-positive NSCLC [15]. A total of 1942 patients were screened for enrolment; 1653 had evaluable samples and 500 (30.2\%) patients had tumors with PD-L1 expression $\geq 50 \%$. A total of 305 patients who met the inclusion criteria were randomized to pembrolizumab (200 $\mathrm{mg}$ every 3 weeks for up to 35 cycles or until documented progressive disease) versus four to six cycles of standard of care platinum-based chemotherapy (platinum/pemetrexed, platinum/gemcitabine, or carboplatin/paclitaxel) as firstline treatment. Pemetrexed maintenance therapy was received by $30 \%$ of patients with non-squamous histology. In addition, $43.7 \%$ of patients in the control arm crossed over per protocol to pembrolizumab upon disease progression. Patients were excluded from the trial if they were harboring EGFR mutations or ALK translocations, had an Eastern Cooperative Oncology Group (ECOG) performance status $\geq 2$, had untreated brain metastasis, or were receiving any dose of oral steroids for an autoimmune disease. The primary endpoint of the trial was the median PFS. Compared with standard first-line platinum-based chemotherapy, pembrolizumab significantly improved the primary endpoint from 6.0 to 10.3 months (hazard ratio (HR), 0.50; 95\% confidence interval (CI), 0.37-0.68; $P<$ $0.001)$. The RR according to the Response Evaluation Criteria In Solid Tumors (RECIST) (44.8\% vs. $27.8 \%$; $P<0.001$ ) and OS (not reached in both arms; HR, 0.60; 95\% CI, 0.41-0.89; $P=0.005$ ) were also improved, with a 1 -year OS of $70 \%$ versus $54 \%$ [15]. The benefit of pembrolizumab with respect to PFS was evident in all subgroups examined according to sex, age, histology, smoking status, and brain metastases at baseline. However, the benefit was lower in female and never-smoker patients (probably related to the lower mutational load in this population [16]), and the greatest benefit to PFS was observed in patients with squamous histology ( $\mathrm{HR}, 0.35 ; 95 \% \mathrm{CI}, 0.17-0.71)$. Grade 3, 4, or 5 treatment-related AEs also favored pembrolizumab (26.6\% vs. $53.3 \%$ ). The incidence of grade $3-4$ immune-mediated AEs was 9.7\% with pembrolizumab [15]. Pembrolizumab had a clinically meaningful improvement in quality of life compared to platinum-based chemotherapy [17] (Table 1).

The magnitude of benefit in the control arm was consistent with historic controls [18], suggesting that pembrolizumab efficacy is not overestimated for an ineffective control arm. However, it is unknown whether the survival benefit was due to pembrolizumab treatment being intrinsically more potent as a first-line treatment or because crossover was limited to $<50 \%$ of the patients in the control arm. Indeed, trials in patients with EGFR-mutant or $A L K$-rearranged NSCLC have had much higher rates of crossover from chemotherapy to personalized treatment after platinum-based chemotherapy progression $(65 \%$ in EGFR-mutant [19] and 70\% in ALK-positive populations [20]), leading to a lack of survival differences between treatment arms. The clear benefit for OS could also be due to a potentially lower efficacy of pembrolizumab in platinum-pretreated patients than in chemo-naïve patients, whereas, in the same settings, targeted therapies yield the same benefit $[21,22]$.

In the KEYNOTE-024 trial, $11.7 \%$ of patients in the pembrolizumab arm had previously treated brain metastases at baseline; the PFS benefit in this subgroup was similar to 
Table 1 Immune checkpoint inhibitors in first-line treatment in advanced non-small cell lung cancer patients

\begin{tabular}{|c|c|c|c|c|c|c|}
\hline Study & Phase & $n$ & RR (\%) & PFS (months) & OS (months) & AEs $\geq$ grade $3(\%)$ \\
\hline $\begin{array}{l}\text { KEYNOTE } 024 \text { [15] } \\
\text { Pembrolizumab vs. CT }\end{array}$ & III & $\begin{array}{l}305 \\
\text { PD-L1 } \geq 50 \%\end{array}$ & $\begin{array}{l}44.8 \text { vs. } 27.8 \\
P<0.001\end{array}$ & $\begin{array}{l}10.3 \text { vs. } 6.0 \\
\text { HR, } 0.50 ; P<0.001\end{array}$ & $\begin{array}{l}\text { HR, } 0.60 ; P=0.005 \\
1 \text {-year OS: } 70 \% \text { vs. } 54 \%\end{array}$ & 26.6 vs. 53.3 \\
\hline $\begin{array}{l}\text { CheckMate } 026 \text { [31] } \\
\text { Nivolumab vs. CT }\end{array}$ & III & $\begin{array}{l}423 \\
\text { PD-L1 } \geq 5 \%\end{array}$ & 26.1 vs. 33.5 & $\begin{array}{l}4.2 \text { vs. } 5.9 \\
\text { HR, } 1.15 ; P=0.251\end{array}$ & $\begin{array}{l}\text { HR, } 1.02 \\
14.4 \text { vs. } 13.2 \text { months }\end{array}$ & 17.6 vs. 50.6 \\
\hline $\begin{array}{l}\text { KEYNOTE } 021[39] \\
\text { Pembrolizumab + CT vs. CT }\end{array}$ & $\|$ & 123 & $\begin{array}{l}55 \text { vs. } 29 \\
P=0.0016\end{array}$ & $\begin{array}{l}13.0 \text { vs. } 8.9 \\
H R, 0.53 ; P=0.0102\end{array}$ & $\mathrm{HR}, 0.90 ; P=0.39$ & 39 vs. 26 \\
\hline $\begin{array}{l}\text { CheckMate } 012 \text { [42] } \\
\text { Nivolumab + Ipilimumab/12 weeks } \\
\text { until disease progression or toxicity } \\
\text { Nivolumab + Ipilimumab/6 weeks until } \\
\text { disease progression or toxicity }\end{array}$ & । & $\begin{array}{l}38 \\
40\end{array}$ & $\begin{array}{l}47 \\
38\end{array}$ & $\begin{array}{l}8.1 \\
3.9\end{array}$ & Not calculated & $\begin{array}{l}37 \\
33\end{array}$ \\
\hline
\end{tabular}

$C T$ chemotherapy, $R R$ response rate, PFS progression-free survival, OS overall survival, $A E s$ adverse events

${ }^{a}$ Expression in $\geq 50 \%$ of tumor cells, regardless of the staining intensity with the $22 \mathrm{C} 3$ clone

${ }^{\mathrm{b}}$ Tumor cell membrane staining any intensity $>1 \%$ with the $28-8$ clone Epitomics

that in patients without such metastases at baseline (HR, 0.55 vs. HR, 0.50). The efficacy of pembrolizumab in patients with PD-L1-positive ( $>1 \%)$ NSCLC with untreated or progressive asymptomatic brain metastases measuring between 5 and $20 \mathrm{~mm}$ in diameter has also recently been tested in a phase II trial that reported a cerebral response rate of $33 \%$ [23]; the median duration of confirmed brain responses was 6 months. Approximately $17 \%$ of NSCLC patients have brain metastases at baseline [24]. In our opinion, supra-tentorial asymptomatic brain metastases should not be considered exclusion criteria for immune checkpoint inhibitor treatment. The risk of brain metastases increases over time due to the prolonged survival of patients with advanced NSCLC [25]. Therefore, further investigations are needed to determine optimal treatment combinations with brain radiotherapy, sequences of treatment, and safety [26].

Globally, pembrolizumab results from KEYNOTE-024 [15] were consistent with the efficacy observed in the KEYNOTE-001 study [27] in the subgroup of chemo-naïve patients. The FDA approved pembrolizumab in the firstline setting in this population on October 24, 2016, and on December 15, 2016, the EMA Committee for Medicinal Products for Human Use also approved pembrolizumab as monotherapy in the first-line setting of metastatic NSCLC in adults whose tumors express PD-L1 with a tumor proportion score (TPS) $\geq 50 \%$ and who have no EGFR- or $A L K$-positive tumor mutations. The efficacy of pembrolizumab as a first-line treatment in NSCLC patients with PD-L1 expression $<50 \%$ remains unknown. The ongoing phase III KEYNOTE-042 study (NCT02220894) will assess the survival benefit of pembrolizumab over standard first-line platinum-based chemotherapy in treatment-naïve patients who have tumors with $\geq 1 \%$ PD-L1 positivity. Stratification according to PD-L1 expression (strong ( $\geq$ $50 \%)$ vs. weak (1-49\%)) will be performed in the study.

Among the $30 \%$ of patients whose tumors express PDL1 with a TPS $\geq 50 \%$, other clinical exclusion criteria limit the extended use of pembrolizumab in the first-line setting; for example, exclusion of patients considered unfit or with poor performance status (representing almost 34\% of NSCLC patients in contemporary cohorts [28]), patients with EGFR-mutant and $A L K$-rearranged tumors (approximately $17 \%$ of adenocarcinoma lung cancers in Caucasian populations [29]), and the absence of steroids or autoimmune disorders $(13.5 \%$ of lung cancer patients [30]). As such, the pool of patients eligible for upfront pembrolizumab is certainly not $30 \%$ of all chemo-naïve patients with NSCLC (which represents the percentage of frontline patients whose tumors express PD-L1 with a TPS $\geq 50 \%$ ), but probably closer to $10 \%$; this pool clearly needs to be enlarged.

Moreover, the turnaround time from patient selection to treatment, based on PD-L1 expression, is not reported in KEYNOTE-024 but is expected to be considerablylonger than 1 month. There is a high probability that patients with relatively indolent disease were favored for inclusion in the study, adding another bias compared to routine practice. Patients with a poorer prognosis need to be explored such as in the ongoing phase II trial NCT02879617 evaluating first-line durvalumab in performance status 2 patients with advanced NSCLC.

\section{Nivolumab}

The phase III CheckMate 026 trial tested the efficacy of nivolumab compared to standard first-line chemotherapy (platinum/pemetrexed, platinum/gemcitabine, or carboplatin/paclitaxel) in 423 patients with PD-L1-positive ( $\geq 5 \%$ of expression by $28-8$ clone) advanced NSCLC [31]. Patients harboring EGFR mutations or ALK translocations were ineligible. Patients with adequately treated brain metastases were allowed. No imbalances were reported in either arm regarding brain metastases $(\sim 12 \%)$, histology ( $24 \%$ of squamous), ECOG performance status ( 30\% PS0), or current smokers ( $20 \%$ in both arms). A higher proportion of females was included in the chemotherapy arm $(45.2 \%$ vs. $32.1 \%)$. Maintenance treatment was prescribed in $38 \%$ of patients [31]. No benefit was seen with nivolumab 
compared to chemotherapy in terms of the primary endpoints PFS (4.2 vs. 5.9 months; HR, 1.15; 95\% CI, 0.91$1.45 ; P=0.251)$, OS (14.4 vs. 13.2 months; HR, 1.02; 95\% CI, $0.80-1.30)$, or RR (26.1\% vs. $33.5 \%)$. However, the toxicity profile favored nivolumab, with $17.6 \%$ of patients having grade 3-4. AEs compared to $50.6 \%$ in the chemotherapy arm (Table 1). Of note, patients with NSCLC with strong PD-L1 expression (TPS $\geq$ $50 \%)$ did not derive a greater benefit from nivolumab than those with weaker expression. Nivolumab was the post-discontinuation treatment in $60 \%$ of patients in the chemotherapy arm. The lack of survival benefit could be related to various hypothetical factors. First, there was a higher proportion of tumors with strong PD-L1 expression (TPS $\geq 50 \%$ ) in the control arm compared to the nivolumab arm $(74.1 \%$ vs. $53.2 \%)$. Second, only $44 \%$ of patients in the nivolumab arm received second-line treatment, mostly platinum-based chemotherapy, suggesting that a certain subgroup of patients was untreated [31]. This could be a consequence of hyper-progressive diseases on immunotherapy, as recently reported by Champiat et al. [32]. Overall, results from CheckMate 026 in the whole population and for those with strongly positive PD-L1-expressing tumors are inconsistent with first-line nivolumab performance in phase I/II trials [33].

Although the reason for the contrasting results between the KEYNOTE-024 [15] and Checkmate 026 [31] trials remains unclear, we should consider the nivolumab trial as negative, and we believe that differences in patient selection are the primary cause of this discrepancy. Differences in biomarker tests and in PD-L1 expression cut-off point $(22 \mathrm{C} 3$ and $50 \%$ with pembrolizumab vs. $28-8$ clone and $5 \%$ with nivolumab) could have contributed to the discordant results between the trials. Thus, patients with strong PD-L1 positivity in the KEYNOTE-024 trial may not be similar to patients with strong PD-L1 positivity in the CheckMate 026 trial since the sensitivity of the relevant clones used to define PD-L1 status is potentially different. Additionally, PD-L1 testing was performed after metastatic diagnosis in the pembrolizumab trial, whereas in the nivolumab trial, it was performed in archival tissue biopsy specimens taken within 6 months prior to randomization. However, in the KEYNOTE-010 trial, survival benefit with pembrolizumab as a second-line treatment was independent of whether the PD-L1 test was performed in an archival or in a new tissue biopsy specimen [11]. Further, the efficacy of immune checkpoint inhibitors is higher among smokers [16]; a higher percentage of never-smoker patients was included in the nivolumab trial than in the pembrolizumab trial $(11 \%$ vs. $3 \%)$ and such patients have lower mutational loads that negatively correlate with the success of immune checkpoint-targeting therapies [34]. Another major difference between the trials was the percentage of patients who received radiotherapy prior to enrollment; this percentage was abnormally high (37.6\%) for patients enrolled in the CheckMate 026 trial [31], whereas in the KEYNOTE-024 trial [15], prior radiation therapy of $>30$ Gy within 6 months of the first dose of trial treatment constituted an exclusion criterion. Therefore, sites that were involved in both trials may have operationally favored enrollment of all previously irradiated patients into CheckMate 026. It is clear that previous radiotherapy can have major consequences on the tumor microenvironment [35] and potentially lead to decreased activity of immune checkpoint inhibitors in previously irradiated areas. At this stage, it is unknown which areas (mediastinum, others) were previously irradiated in patients enrolled in the CheckMate 026 trial [31].

Other first-line randomized phase III clinical trials are testing anti-PD-1 monotherapy, such as nivolumab in CheckMate 227 (NCT02477826), or anti-PD-L1 monotherapies such as atezolizumab in IMpower 110 (NCT02409342) and avelumab in the ongoing JAVELIN Lung 100 trial (NCT02576574). These trials may validate immune checkpoint inhibitors as a first-line treatment in patients with PD-L1-positive NSCLC.

\section{Combination of anti-PD-1 antibodies with chemotherapy}

A large body of preclinical data has shown that chemotherapy and radiation modulate the immune response against tumors [36] and that chemotherapy can induce PD-L1 expression in tumor cells $[37,38]$. This has led to clinical investigation of combinations of immune checkpoint inhibitors and chemotherapy.

The phase II KEYNOTE-021 trial $(n=123)$ compared pembrolizumab $200 \mathrm{mg}$ for 2 years concomitant with four cycles of carboplatin-pemetrexed chemotherapy followed by pemetrexed as maintenance therapy with chemotherapy alone [39]. A higher proportion of never-smoker patients ( $25 \%$ vs. $14 \%$ ) and patients with adenocarcinoma histology ( $97 \%$ vs. $87 \%$ ) were included in the pembrolizumab arm. A total of $32 \%$ of patients in the chemotherapy group crossed over to receive pembrolizumab monotherapy as allowed by the study protocol. The combination arm had improved RR (55\% vs. $29 \%$; $P=0.0016$, with $80 \%$ RR among strongly PDL1-positive tumors) and PFS (13.0 vs. 8.9 months; HR, 0.53; 95\% CI, $0.31-0.91 ; P=0.010$ ) compared to chemotherapy alone; however, the frequency of grade 3-4 treatmentrelated AEs was higher in the concomitant arm (39\% vs. 26\%) (Table 1). Of note, the chemotherapy arm also demonstrated impressive PFS, suggesting a high level of patient selection. Indeed, median PFS was much longer than in similar populations included in other trials with the same chemotherapy schedule, for example, the PointBreak trial (5.6 months) [40]. The KEYNOTE-021 study reported a shorter time to response in the combination arm compared 
to the chemotherapy alone arm (1.5 vs. 2.7 months), suggesting this approach could be a good strategy for symptomatic patients [39]. These results are similar to those previously reported in phase I trials, suggesting that combination treatment could be an optimal strategy.

Overall, while very promising, these results need to be validated in a phase III trial. The preliminary RR of $80 \%$ in patients with tumors harboring PD-L1 expression $\geq 50 \%$ treated with the combination therapy appears intriguing, but numbers are too small to draw any definitive conclusions. The ongoing phase III trials KEYNOTE-189 (NCT02578680) and KEYNOTE-407 (NCT02775435) with pembrolizumab and the IMpower 132 (NCT02657434), IMpower 130 (NCT02367781), IMpower 131 (NCT02367794), and IMpower 150 (NCT02 366143) trials with atezolizumab are testing combination treatment versus standard of care and could help clarify the best treatment strategy for this population.

\section{Other open questions in the first-line setting}

The third approach to position immunotherapy in the first-line setting is the combination of PD-1/PD-L1 blockade with anti-cytotoxic T-lymphocyte-associated protein 4 (CTLA-4) compounds (Fig. 1). Early preclinical studies have suggested that combined CTLA-4 and PD-1 pathway blockade produces synergistic anti-tumor activity
[5, 41], providing the rationale for clinical studies. The high efficacy of nivolumab plus ipilimumab has been recently reported in PD-L1-positive tumors (Table 1) [42]. The phase III CheckMate 227 (NCT02477826) trial with nivolumab plus ipilimumab, and the MYSTIC (NCT02453282) and NEPTUNE (NCT02542293) trials with durvalumab plus tremelimumab are comparing this strategy to anti-PD-1/PD-L1 monotherapy or chemotherapy. The toxicity profiles of these combinations might, however, limit their applicability.

The optimal strategy for NSCLC patients with tumor PD-L1 expression $<50 \%$ has to be better defined - potential candidate therapies include concomitant treatments with chemotherapy, a combination of immune checkpoint inhibitors, or sequential strategies. This issue is important because of the limited standard second-line options currently available $[43,44]$ in cases in which immune checkpoint inhibitors are prescribed as the first-line treatment.

Treatment duration with immune checkpoint inhibitors and economic costs are also important issues. Therefore, detailed health economic analyses are required to avoid inequities in access to these treatments [45]. New tools should be applied, such as the ESMO Magnitude of Clinical Benefit Scale (ESMO-MCBS), which uses a rational, structured, and consistent approach to derive a

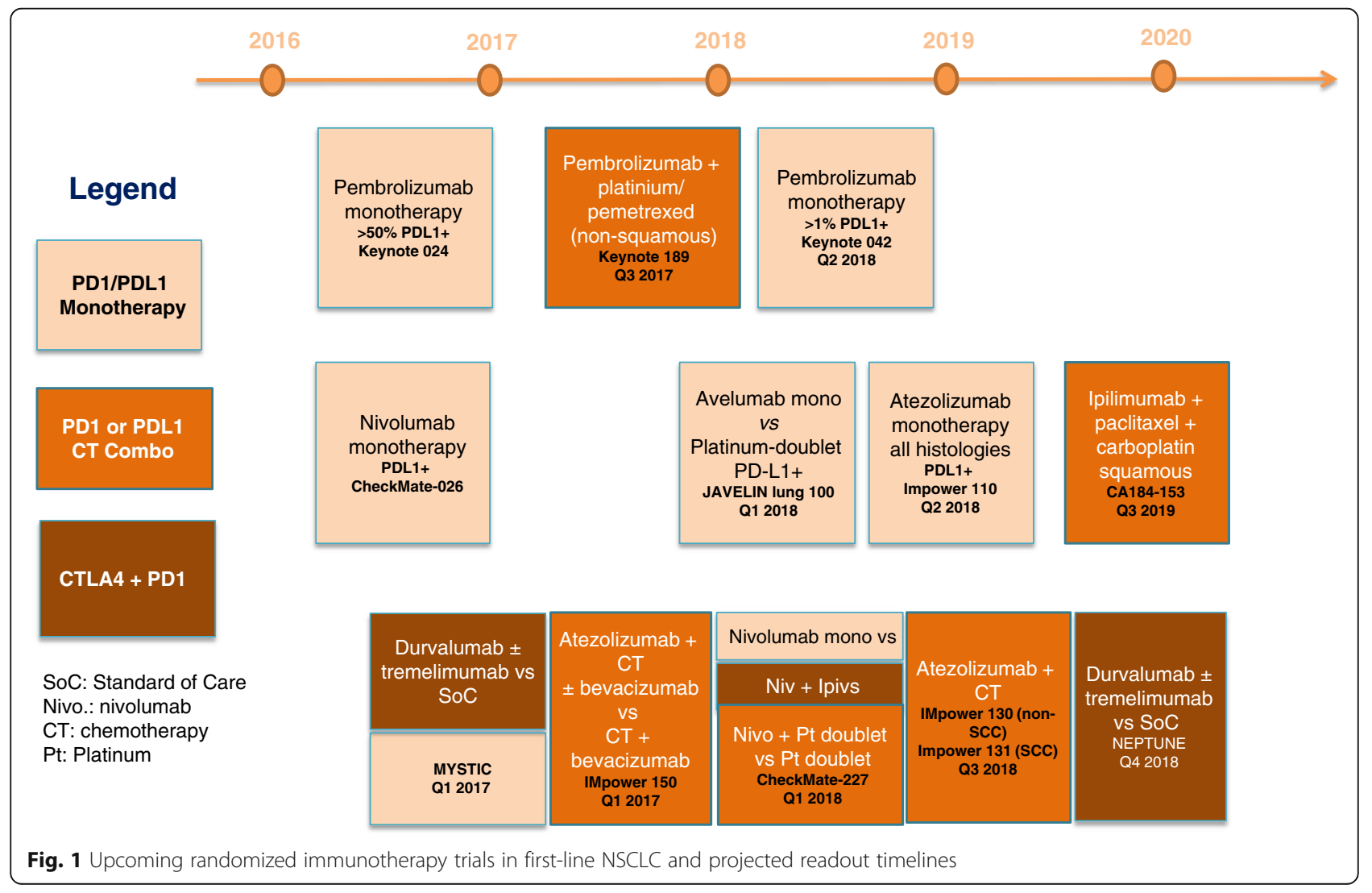


relative ranking of the magnitude of clinically meaningful benefit that can be expected from new anti-cancer therapies $[46,47]$.

\section{Conclusions}

Immune checkpoint inhibitors are the standard of care for second-line treatment in advanced NSCLC, and pembrolizumab should be considered a standard first-line treatment in NSCLC patients with a good performance status whose tumors have PD-L1 expression $\geq 50 \%$. PD-L1 status determined by immunohistochemistry should be considered a reflex biomarker, along with EGFR mutation and $A L K$ translocation, in guiding treatment of front-line patients with advanced NSCLC.

Discrepancies in patient selection (notably, previous radiotherapy) and PD-L1 testing methods could explain the negative results achieved with nivolumab in the first-line setting. Better outcomes were observed with chemotherapy combined with pembrolizumab compared to chemotherapy alone in a small, randomized phase II trial. Whether this strategy is better than immunotherapy alone or the combination of different checkpoint inhibitors remains unknown since no phase III trial data is yet available.

\section{Acknowledgments}

None.

\section{Authors' contributions}

$\mathrm{JR}, \mathrm{BB}$, and JCS wrote and corrected the manuscript. All authors read and approved the final manuscript.

\section{Competing interests}

JCS reports consultancy fees from AstraZeneca, Astex, Clovis, GSK, Gammamabs, Lilly, MSD, Mission Therapeutics, Merus, Pfizer, Pharmamar, Pierre Fabre, Roche-Genentech, Sanofi, Servier, Symphogen, and Takeda. The other authors declare no competing interests.

\section{Publisher's Note}

Springer Nature remains neutral with regard to jurisdictional claims in published maps and institutional affiliations.

\section{Author details}

${ }^{1}$ Cancer Medicine Department, Gustave Roussy, Villejuif, France. ${ }^{2}$ Medical Oncology Department, Hospital Vall d'Hebron, Barcelona, Spain. ${ }^{3}$ University Paris-Sud, Orsay, France.

Received: 3 January 2017 Accepted: 16 February 2017

Published online: 13 March 2017

\section{References}

1. Midha A, Dearden S, McCormack R. EGFR mutation incidence in non-smallcell lung cancer of adenocarcinoma histology: a systematic review and global map by ethnicity (mutMapll). Am J Cancer Res. 2015;5:2892-911.

2. Dearden S, Stevens J, Wu Y-L, Blowers D. Mutation incidence and coincidence in non small-cell lung cancer: meta-analyses by ethnicity and histology (mutMap). Ann Oncol. 2013:24:2371-6. doi:10.1093/annonc/mdt205.

3. Novello S, Barlesi F, Califano R, Cufer T, Ekman S, Levra MG, Kerr K, Popat S, Reck M, Senan S, Simo GV, Vansteenkiste J, Peters S, ESMO Guidelines Committee. Metastatic non-small-cell lung cancer: ESMO Clinical Practice Guidelines for diagnosis, treatment and follow-up. Ann Oncol. 2016;27:v1-v27. doi:10.1093/annonc/mdw326.
4. Topalian SL, Drake CG, Pardoll DM. Immune checkpoint blockade: a common denominator approach to cancer therapy. Cancer Cell. 2015;27: 450-61. doi:10.1016/j.ccell.2015.03.001

5. Pardoll DM. The blockade of immune checkpoints in cancer immunotherapy. Nat Rev Cancer. 2012;12:252-64. doi:10.1038/nrc3239.

6. Hanahan D, Weinberg RA. Hallmarks of cancer: the next generation. Cell. 2011;144:646-74. doi:10.1016/j.cell.2011.02.013

7. Taube JM, Klein A, Brahmer JR, Xu H, Pan X, Kim JH, et al. Association of PD-1, PD-1 ligands, and other features of the tumor immune microenvironment with response to anti-PD-1 therapy. Clin Cancer Res. 2014;20:5064-74. doi:10.1158/1078-0432.CCR-13-3271.

8. Jiang Y, Li Y, Zhu B. T-cell exhaustion in the tumor microenvironment. Cell Death Dis. 2015;6:e1792. doi:10.1038/cddis.2015.162.

9. Brahmer J, Reckamp KL, Baas P, Crinò L, Eberhardt WEE, Poddubskaya E, et al. Nivolumab versus docetaxel in advanced squamous-cell non-small-cell lung cancer. N Engl J Med. 2015;373:123-35. doi:10.1056/NEJMoa1504627.

10. Borghaei H, Paz-Ares L, Horn L, Spigel DR, Steins M, Ready NE, et al. Nivolumab versus docetaxel in advanced nonsquamous non-small-cell lung cancer. N Engl J Med. 2015:373:1627-39. doi:10.1056/NEJMoa1507643.

11. Herbst RS, Baas P, Kim DW, Felip E, Pérez-Gracia JL, Han JY, et al. Pembrolizumab versus docetaxel for previously treated, PD-L1-positive, advanced non-small-cell lung cancer (KEYNOTE-010): a randomised controlled trial. Lancet. 2016;387:1540-50. doi:10.1016/S0140-6736(15)01281-7.

12. Rittmeyer A, Barlesi F, Waterkamp D, Park K, Ciardiello F, von Pawel J, OAK Study Group, et al. Atezolizumab versus docetaxel in patients with previously treated non-small-cell lung cancer (OAK): a phase 3, open-label, multicentre randomised controlled trial. Lancet. 2017;389:255-65. doi:10. 1016/S0140-6736(16)32517-X

13. Pillai RN, Behera M, Owonikoko TK, Kamphorst AO, Pakkala S, Belani CP, et al. Evaluation of toxicity profile of PD-1 versus PD-L1 inhibitors in nonsmall cell lung cancer. J Thorac Oncol. 2016;11:OA03.06.

14. Gettinger SN, Horn L, Gandhi L, Spigel DR, Antonia SJ, Rizvi NA, et al. Overall survival and long-term safety of nivolumab (anti-programmed death 1 antibody, BMS-936558, ONO-4538) in patients with previously treated advanced non-small-cell lung cancer. J Clin Oncol. 2015;33:2004-12. doi:10.1200/JCO.2014.58.3708.

15. Reck M, Rodríguez-Abreu D, Robinson AG, Hui R, Csőszi T, Fülöp A, KEYNOTE-024 Investigators, et al. Pembrolizumab versus chemotherapy for PD-L1-positive non-small-cell lung cancer. N Engl J Med. 2016;375:1823-33. doi:10.1056/NEJMoa1606774

16. Rizvi NA, Hellmann MD, Snyder A, Kvistborg P, Makarov V, Havel JJ, et al. Cancer immunology. Mutational landscape determines sensitivity to PD1 blockade in non-small cell lung cancer. Science. 2015;348:124-8. doi:10.1126/science.aaa1348.

17. Brahmer J, Rodriguez-Abreu D, Robinson AG, Hui R, Csoszi T, Fulop A, et al. Health-related quality of life for pembrolizumab vs chemotherapy in advanced NSCLC with PD-L1 TPS $\geq 50 \%$ : data from KEYNOTE-024. J Clin Oncol. 2016;34:Abstr 7153.

18. Scagliotti GV, Parikh P, von Pawel J, Biesma B, Vansteenkiste J, Manegold C, et al. Phase III study comparing cisplatin plus gemcitabine with cisplatin plus pemetrexed in chemotherapy-naive patients with advanced-stage non-smallcell lung cancer. J Clin Oncol. 2008;26:3543-51. doi:10.1200/JCO.2007.15.0375.

19. Fukuoka M, Wu Y-L, Thongprasert S, Sunpaweravong P, Leong S-S, Sriuranpong $V$, et al. Biomarker analyses and final overall survival results from a phase III, randomized, open-label, first-line study of gefitinib versus carboplatin/paclitaxel in clinically selected patients with advanced nonsmall-cell lung cancer in Asia (IPASS). J Clin Oncol. 2011;29:2866-74. doi:10.1200/JCO.2010.33.4235.

20. Solomon BJ, Mok T, Kim D-W, Wu Y-L, Nakagawa K, Mekhail T, PROFILE 1014 Investigators, et al. First-line crizotinib versus chemotherapy in ALK-positive lung cancer. N Engl J Med. 2014;371:2167-77. doi:10.1056/NEJMoa1408440.

21. Miyauchi E, Inoue A, Kobayashi K, Maemondo M, Sugawara S, Oizumi S, NorthEast Japan Study Group, et al. Efficacy of chemotherapy after first-line gefitinib therapy in EGFR mutation-positive advanced non-small cell lung cancer-data from a randomized Phase III study comparing gefitinib with carboplatin plus paclitaxel (NEJ002). Jpn J Clin Oncol. 2015:45:670-6. doi:10.1093/jjco/hyv054.

22. Rosell R, Moran T, Queralt C, Porta R, Cardenal F, Camps C, Spanish Lung Cancer Group, et al. Screening for epidermal growth factor receptor mutations in lung cancer. N Engl J Med. 2009;361:958-67. doi:10.1056/NEJMoa0904554.

23. Goldberg SB, Gettinger SN, Mahajan A, Chiang AC, Herbst RS, Sznol M, et al. Pembrolizumab for patients with melanoma or non-small-cell lung cancer 
and untreated brain metastases: early analysis of a non-randomised, openlabel, phase 2 trial. Lancet Oncol. 2016;17:976-83. doi:10.1016/S14702045(16)30053-5.

24. Moro-Sibilot D, Smit E, de Castro Carpeño J, Lesniewski-Kmak K, Aerts JG, Villatoro R, et al. Non-small cell lung cancer patients with brain metastases treated with first-line platinum-doublet chemotherapy: analysis from the European FRAME study. Lung Cancer. 2015;90:427-32. doi:10.1016/j.lungcan. 2015.11.011.

25. Peters $S$, Bexelius $C$, Munk V, Leighl $N$. The impact of brain metastasis on quality of life, resource utilization and survival in patients with non-small-cell lung cancer. Cancer Treat Rev. 2016;45:139-62. doi:10.1016/j.ctrv.2016.03.009.

26. Franceschini D, Franzese C, Navarria P, Ascolese AM, De Rose F, Del Vecchio M, Santoro A, Scorsetti M. Radiotherapy and immunotherapy: can this combination change the prognosis of patients with melanoma brain metastases? Cancer Treat Rev. 2016:50:1-8. doi:10.1016/j.ctrv.2016.08.003.

27. Garon EB, Rizvi NA, Hui R, Leighl N, Balmanoukian AS, Eder JP, KEYNOTE-001 Investigators, et al. Pembrolizumab for the treatment of non-small-cell lung cancer. N Engl J Med. 2015;372:2018-28. doi:10.1056/NEJMoa1501824.

28. Salloum RG, Smith TJ, Jensen GA, Lafata JE. Using claims-based measures to predict performance status score in patients with lung cancer. Cancer. 2011 117:1038-48. doi:10.1002/cncr.25677.

29. Barlesi F, Mazieres J, Merlio JP, Debieuvre D, Mosser J, Lena H, Biomarkers France Contributors, et al. Routine molecular profiling of patients with advanced non-small-cell lung cancer: results of a 1-year nationwide programme of the French Cooperative Thoracic Intergroup (IFCT). Lancet. 2016;387:1415-26. doi:10.1016/S0140-6736(16)00004-0.

30. Khan SA, Pruitt SL, Xuan L, Gerber DE. Prevalence of autoimmune disease among patients with lung cancer: implications for immunotherapy treatment options. JAMA Oncol. 2016;2:1507-8. doi:10.1001/jamaoncol.2016.2238.

31. Socinski M, Creelan B, Horn L, Reck M, Paz-Ares L, Steins M, et al. NSCLC, metastatic CheckMate 026: a phase 3 trial of nivolumab vs investigator's choice of platinum-based doublet chemotherapy as first-line therapy for stage IV/recurrent programmed death ligand 1 (PD-L1)-positive NSCLC. Ann Oncol. 2016;27:LBA7_PR. doi: 10.1093/annonc/mdw435.39.

32. Champiat S, Dercle L, Ammari S, Massard C, Hollebecque A, Postel-Vinay S, et al. Hyperprogressive disease (HPD) is a new pattern of progression in cancer patients treated by anti-PD-1/PD-L1. Clin Cancer Res. 2016. Ahead of print. doi:10.1158/1078-0432.CCR-16-1741.

33. Gettinger S, Rizvi NA, Chow LQ, Borghaei H, Brahmer J, Ready N, et al. Nivolumab monotherapy for first-line treatment of advanced non-small-cell lung cancer. J Clin Oncol. 2016;34:2980-7. doi:10.1200/JCO.2016.66.9929.

34. Champiat S, Ferté C, Lebel-Binay S, Eggermont A, Soria JC. Exomics and immunogenics: bridging mutational load and immune checkpoints efficacy. Oncoimmunology. 2014;3:e27817. doi:10.4161/onci.27817.

35. Leroi N, Lallemand F, Coucke P, Noel A, Martinive P. Impacts of ionizing radiation on the different compartments of the tumor microenvironment. Front Pharmacol. 2016:7:78. doi:10.3389/fphar.2016.00078.

36. Liu WM, Fowler DW, Smith P, Dalgleish AG. Pre-treatment with chemotherapy can enhance the antigenicity and immunogenicity of tumours by promoting adaptive immune responses. Br J Cancer. 2010;102 115-23. doi:10.1038/sj.bjc.6605465.

37. Peng J, Hamanishi J, Matsumura N, Abiko K, Murat K, Baba T, et al. Chemotherapy induces programmed cell death-ligand 1 overexpression via the nuclear factor-kB to foster an immunosuppressive tumor microenvironment in ovarian cancer. Cancer Res. 2015;75:5034-45. doi:10.1158/0008-5472.CAN-14-3098.

38. Zhang P, Ma Y, Lv C, Huang M, Li M, Dong B, et al. The up-regulation of PD-L1 promotes the resistant response in non-small cell lung cancer patients with neoadjuvant chemotherapy. Cancer Sci. 2016;107:1563-71. doi:10.1111/cas.13072.

39. Langer CJ, Gadgeel SM, Borghaei H, Papadimitrakopoulou VA, Patnaik A, Powell SF, KEYNOTE-021 Investigators, et al. Carboplatin and pemetrexed with or without pembrolizumab for advanced, non-squamous non-small-cell lung cancer: a randomised, phase 2 cohort of the open-label KEYNOTE-021 study. Lancet Oncol. 2016;17:1497-508. doi:10.1016/S1470-2045(16)30498-3.

40. Patel JD, Socinski MA, Garon EB, Reynolds CH, Spigel DR, Olsen MR, et al. PointBreak: a randomized phase III study of pemetrexed plus carboplatin and bevacizumab followed by maintenance pemetrexed and bevacizumab versus paclitaxel plus carboplatin and bevacizumab followed by maintenance bevacizumab in patients with stage IIIB or IV nonsquamous non-small-cell lung cancer. J Clin Oncol. 2013;31:4349-57.

41. Curran MA, Montalvo W, Yagita H, Allison JP. PD-1 and CTLA-4 combination blockade expands infiltrating $T$ cells and reduces regulatory $T$ and myeloid cells within B16 melanoma tumors. Proc Natl Acad Sci U S A. 2010;107: 4275-80. doi:10.1073/pnas.0915174107.

42. Hellmann MD, Rizvi NA, Goldman JW, Gettinger SN, Borghaei H, Brahmer JR, et al. Nivolumab plus ipilimumab as first-line treatment for advanced non-small-cell lung cancer (CheckMate 012): results of an open-label, phase 1, multicohort study. Lancet Oncol. 2017;18:31-41. doi:10.1016/S1470-2045(16)30624-6.

43. Garon EB, Ciuleanu TE, Arrieta O, Prabhash K, Syrigos KN, Goksel T, et al. Ramucirumab plus docetaxel versus placebo plus docetaxel for second-line treatment of stage IV non-small-cell lung cancer after disease progression on platinum-based therapy (REVEL): a multicentre, double-blind, randomised phase 3 trial. Lancet. 2014:384:665-73. doi:10.1016/S0140-6736(14)60845-X

44. Reck M, Kaiser R, Mellemgaard A, Douillard J-Y, Orlov S, Krzakowski M, LUME-Lung 1 Study Group, et al. Docetaxel plus nintedanib versus docetaxel plus placebo in patients with previously treated non-small-cell lung cancer (LUME-Lung 1): a phase 3, double-blind, randomised controlled trial. Lancet Oncol. 2014;15:143-55. doi:10.1016/S1470-2045(13)70586-2.

45. Cherny N, Sullivan R, Torode J, Saar M, Eniu A. ESMO European Consortium Study on the availability, out-of-pocket costs and accessibility of antineoplastic medicines in Europe. Ann Oncol. 2016;27:1423-43. doi:10.1093/annonc/mdw213.

46. Cherny NI, Sullivan R, Dafni U, Kerst JM, Sobrero A, Zielinski C, de Vries EGE, Piccart MJ. A standardised, generic, validated approach to stratify the magnitude of clinical benefit that can be anticipated from anti-cancer therapies: the European Society for Medical Oncology Magnitude of Clinical Benefit Scale (ESMO-MCBS). Ann Oncol. 2015;26:1547-73. doi:10.1093/annonc/mdv249.

47. Cherny NI, Sullivan R, Dafni U, Kerst JM, Sobrero A, Zielinski C, et al. ESMO Magnitude of Clinical Benefit Scale V.1.0 questions and answers. ESMO Open. 2016;1:e000100. doi:10.1136/esmoopen-2016-000100.

\section{Submit your next manuscript to BioMed Central and we will help you at every step:}

- We accept pre-submission inquiries

- Our selector tool helps you to find the most relevant journal

- We provide round the clock customer support

- Convenient online submission

- Thorough peer review

- Inclusion in PubMed and all major indexing services

- Maximum visibility for your research

Submit your manuscript at www.biomedcentral.com/submit
) Biomed Central 